\title{
OPTIMAL EXPERIMENTAL DESIGN FOR AN INVERSE SCATTERING PROBLEM
}

\author{
Raghuram Rangarajan, Raviv Raich, and Alfred O. Hero III \\ Department of Electrical Engineering and Computer Science, University of Michigan \\ \{rangaraj, ravivr, hero\}@eecs.umich.edu
}

\begin{abstract}
We consider the problem of imaging a medium using an array of sensors. More specifically, we are interested in optimally designing a sequence of experiments for probing a medium in order to form an image of the scatterers present in the medium. In this paper, we consider the case where the received signal is corrupted by noise. We derive an expression for the mean square error for estimating the scatter coefficients and find the optimal sequence scheme that minimizes this error. Using the expression for the minimum mean square error, we show that we can do better than any beamforming approach to imaging. In the process, we also find the optimal energy allocation between the sequence of experiments. Closed-form expressions for the optimal transmission scheme and the minimum mean square error are provided.
\end{abstract}

\section{INTRODUCTION}

The problem of imaging a medium using an array of transducers has been widely studied in a variety of research areas such as mine detection, ultrasonic medical imaging, foliage penetrating radar, non-destructive testing [1], and active audio. A recent approach uses the concept of time reversal, which works by exploiting the reciprocity of a physical channel, e.g., acoustic, optical, or radio-frequency. One implication of reciprocity is that a receiver can reflect back a time reversed signal, thereby focusing the signal at the transmitter source [2]. Furthermore, with suitable prefiltering and aperture, the signal energy can also be focused on an arbitrary spatial location.

In this paper, we set up the problem of optimally designing a sequence of measurements to image a medium of multiple scatterers using an array of transducers under a near field approximation of the scatterers in the medium. The probing method uses an array of trans-

This research was partially supported by ARO-DARPA MURI Grant \#DAAD19-02-1-0262 ducers, e.g., antennas, that both illuminate and measure the backscattered signal field. The method consists of the following four signal processing steps at the transducer array: (i) transmission of time varying signals into the medium; (ii) recording of the backscattered field from the medium; (iii) retransmission into the medium of a spatially filtered version of the recorded backscatter signals; (iv) measurement and spatial filtering of the backscattered signals.

We address the problem of optimally setting up a set of experiments $[3,4]$ that takes advantage of past measurements in order to improve the performance of scattering coefficients estimation. After obtaining the past measurements, we can offer a method of sounding the channel that alters the statistics of the next measurement to yield an overall reduction in MSE.

In this paper, we systematically study the effect of receiver noise on the imaging performance. We evaluate the imaging performance through the mean square error of the maximum likelihood estimates of the scatter coefficients. We obtain a closed-form expression for the MSE for the optimal transmission scheme for the single scatterer case. We provide a suboptimal solution, which outperforms the conventional beamforming solution. We also find the optimal energy allocation scheme between the two transmissions involved in steps (i) and (iii). The case of multiple scatterers has the same formulation but that, unlike the single scatterer case studied here, a closed-form minimum MSE solution for the optimal sequence of transmissions appears intractable.

In Section 2, we present the concept of imaging a medium using an iterative process of array measurements. In Section 3, we formulate the MSE criterion for maximum likelihood estimation of the scatterer coefficients. We also offer an optimal transmission scheme that minimizes the MSE taking advantage of past measurements. In Section 4, we provide simulation results to verify the optimal solution and present a suboptimal scheme that outperforms the conventional beamformer. We conclude this paper in Section 5. 


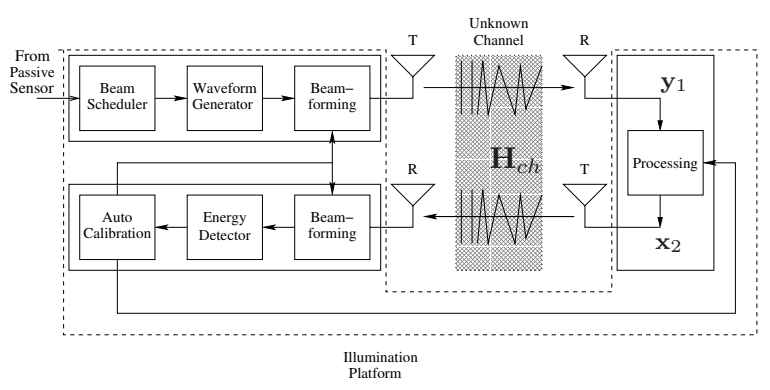

Fig. 1. Measurement setup

\section{MODEL AND MATHEMATICAL DESCRIPTION}

The block diagram in Fig. 1 provides a high level description of the system. The signal flow in the block diagram is read clockwise from the upper left corner of the diagram. The three blocks surrounded by the box on the upper left of the diagram incorporate voxel selection (beam scheduling), spatio-temporal waveform selection and beamsteering followed by transmission into the medium, denoted as a dispersive spatio-temporal channel function $\mathbf{H}_{c h}$. The block on the right of the diagram processes the received backscattered signal and reinserts it into the medium $\mathbf{H}_{c h}$. The third block on the lower left of the diagram can be used to extract a signal which will enable us to calibrate the sensor locations when they are unknown.

We have $N$ transducers located at positions $\left\{\mathbf{r}_{k}^{a}\right\}_{k=1}^{N}$, that transmit narrowband signals with center frequency $\omega \mathrm{rad} / \mathrm{sec}$. We assume there is an imaging area (or volume) divided into $V$ voxels at positions $\left\{\mathbf{r}_{k}^{v}\right\}_{k=1}^{V}$ which we want to image. The channel, denoted $\mathbf{h}_{i}$, between a candidate voxel $i$ and the $N$ transducers is assumed to take the form,

$$
\mathbf{h}_{i}=\left[\left(\frac{\exp \left(-j \omega / c\left\|\mathbf{r}_{k}^{a}-\mathbf{r}_{i}^{v}\right\|\right)}{\left\|\mathbf{r}_{k}^{a}-\mathbf{r}_{i}^{v}\right\|}\right)_{k=1 \ldots N}\right]^{T} .
$$

This channel model is a narrowband near-field approximation, which ignores the effect of multiple scattering and has been widely adopted in other scattering studies, e.g., [5]. Each voxel can be characterized by its scatter coefficient, e.g., radar cross-section (RCS), $\left\{d_{v}\right\}_{v=1}^{V}$, which indicates the proportion of the received field that is re-radiated. Thus the channel between the transmitted field and the measured backscattered field at the transducer array is,

$$
\begin{aligned}
\mathbf{H}_{c h} & =\mathbf{H D H}^{T} \\
\mathbf{H} & =\left[\mathbf{h}_{1}, \mathbf{h}_{2}, \cdots, \mathbf{h}_{V}\right] \\
\mathbf{D} & =\operatorname{diag}(\mathbf{d}) ; \quad \mathbf{d}=\left[d_{1}, \ldots, d_{V}\right]^{T} .
\end{aligned}
$$

Note $\mathbf{H}$ is $N \times V, \mathbf{D}$ is $V \times V$, and $\mathbf{H}_{c h}$ is $N \times N$ matrix.

The probing mechanism for imaging of the scatter cross section follows a four step process, generating the following sequence of noise contaminated signals.

Step 1: The transducers first send $\mathbf{x}_{1}$, in the direction of the beam steering vector $\mathbf{v}$, i.e., $\mathbf{x}_{1}=\mathbf{v}$.

Step 2: Next, the transducers receive the backscattered signal plus noise $\mathbf{n}_{1}$, after it has traveled through the channel $\mathbf{H}_{c h}$ :

$$
\mathbf{y}_{1}=\mathbf{H}_{c h} \mathbf{x}_{1}+\mathbf{n}_{1}=\mathbf{H D H}^{T} \mathbf{v}+\mathbf{n}_{1} .
$$

Step 3: The transducers transmit $\mathbf{x}_{2}=\mathbf{s}\left(\mathbf{y}_{1}\right)$ which in general, is a function of $\mathbf{y}_{1}$ allowing the system to take advantage of the knowledge of the received signal.

Step 4: Finally, the transducers receive the second backscattered signal $\mathbf{y}_{2}$.

$$
\mathbf{y}_{2}=\mathbf{H}_{c h} \mathbf{x}_{2}+\mathbf{n}_{2}=\mathbf{H D H}^{T} \mathbf{s}\left(\mathbf{y}_{1}\right)+\mathbf{n}_{2} .
$$

The noises $\mathbf{n}_{1}, \mathbf{n}_{2}$ are i.i.d complex normal random vectors with zero mean and a covariance matrix $\sigma^{2} \mathbf{I}$.

\section{ERROR ANALYSIS AND MSE MINIMIZATION}

Equations (2) and (3) can be rewritten in the form,

$$
\begin{aligned}
& \mathbf{y}_{1}=\mathbf{L}_{1} \mathbf{d}+\mathbf{n}_{1} \\
& \mathbf{y}_{2}=\mathbf{L}_{2} \mathbf{d}+\mathbf{n}_{2},
\end{aligned}
$$

where $\mathbf{L}_{1}=\mathbf{H} \operatorname{diag}\left(\mathbf{H}^{T} \mathbf{v}\right), \quad \mathbf{L}_{2}=\mathbf{H} \operatorname{diag}\left(\mathbf{H}^{T} \mathbf{s}\left(\mathbf{y}_{1}\right)\right)$. Since the measurements $\mathbf{y}_{1}$ and $\mathbf{y}_{2}$ are linear in $\mathbf{d}$ and Gaussian, the maximum likelihood estimate of $\mathbf{d}$ is

$$
\hat{\mathbf{d}}=\left(\mathbf{L}_{1}^{H} \mathbf{L}_{1}+\mathbf{L}_{2}^{H} \mathbf{L}_{2}\right)^{-1}\left(\mathbf{L}_{1}^{H} \mathbf{y}_{1}+\mathbf{L}_{2}^{H} \mathbf{y}_{2}\right) .
$$

The mean square error [6] would then be,

$$
\begin{aligned}
\mathrm{MSE}= & \mathrm{E}\left[\|\hat{\mathbf{d}}-\mathbf{d}\|^{2}\right]=\mathrm{E}_{\mathbf{y}_{1}}\left[\mathrm{MSE} \mid \mathbf{y}_{1}\right] \\
\mathrm{MSE} \mid \mathbf{y}_{1}= & \mathrm{E}_{\mathbf{y}_{2} \mid \mathbf{y}_{1}}\left[\|\hat{\mathbf{d}}-\mathbf{d}\|^{2}\right]=\left\{\left(\mathbf{L}_{1}^{H} \mathbf{L}_{1}+\mathbf{L}_{2}^{H} \mathbf{L}_{2}\right)^{-1}\right. \\
& \left.\left(\mathbf{L}_{1}^{H} \mathbf{n}_{1} \mathbf{n}_{1}^{H} \mathbf{L}_{1}+\mathbf{L}_{2}^{H} \mathbf{L}_{2} \sigma^{2}\right)\left(\mathbf{L}_{1}^{H} \mathbf{L}_{1}+\mathbf{L}_{2}^{H} \mathbf{L}_{2}\right)^{-1}\right\},
\end{aligned}
$$

where $\mathbf{L}_{1}$ and $\mathbf{L}_{2}$ are matrices, and finding the optimal $\mathbf{s}\left(\mathbf{y}_{1}\right)$ that minimizes the MSE is not straightforward. To get an idea of the solution minimizing the MSE, let us consider the single scatterer case.

For the single scatterer case, equations (4) and (5) can be simplified to

$$
\begin{aligned}
& \mathbf{y}_{1}=\mathbf{l}_{1} d_{1}+\mathbf{n}_{1} \\
& \mathbf{y}_{2}=\mathbf{l}_{2} d_{1}+\mathbf{n}_{2},
\end{aligned}
$$

where $\mathbf{l}_{1}=\mathbf{h}_{1} \mathbf{h}_{1}^{T} \mathbf{v}$ and $\mathbf{l}_{2}=\mathbf{h}_{1} \mathbf{h}_{1}^{T} \mathbf{s}\left(\mathbf{y}_{1}\right)$. 
In the case of a monostatic system, we observe only $\mathbf{y}_{2}$. Since $\mathbf{y}_{2}$ is a non-Gaussian process, the problem becomes complicated. In a bi-static system, where both the signals $\mathbf{y}_{1}$ and $\mathbf{y}_{2}$ are observed, the MSE $\mid \mathbf{y}_{1}$ can be written as

$$
\begin{aligned}
\mathrm{MSE} \mid \mathbf{y}_{1} & =\sigma^{2}\left\{\frac{1}{\left(\mathbf{l}_{1}^{H} \mathbf{l}_{1}+\mathbf{l}_{2}^{H} \mathbf{l}_{2}\right)}-\frac{\mathbf{l}_{1}^{H} \mathbf{l}_{1}\left(1-|\alpha|^{2}\right)}{\left(\mathbf{l}_{1}^{H} \mathbf{l}_{1}+\mathbf{l}_{2}^{H} \mathbf{l}_{2}\right)^{2}}\right\} \\
& =\sigma^{2}\left(\frac{1}{f}-\frac{c}{f^{2}}\right),
\end{aligned}
$$

where $f=\left(\mathbf{l}_{1}^{H} \mathbf{l}_{1}+\mathbf{l}_{2}^{H} \mathbf{l}_{2}\right), c=\mathbf{l}_{1}^{H} \mathbf{l}_{1}\left(1-|\alpha|^{2}\right)$ and $\alpha=$ $\frac{\mathbf{h}_{1}^{H} \mathbf{y}_{1}}{\left\|\mathbf{h}_{1}\right\| \sigma}-\frac{\left\|\mathbf{h}_{1}\right\| d_{1} \mathbf{h}_{1}^{T} \mathbf{v}}{\sigma}$ is complex Gaussian with zero mean and unit variance.

Minimizing the MSE with respect to $\mathbf{s}\left(\mathbf{y}_{1}\right)$ is equivalent to minimizing with respect to $f$. $f$ is a function of $\mathbf{v}$ and $\mathbf{s}$. Since the squared norm of $\mathbf{v}$ and $\mathbf{s}$ is limited to $E_{1}$ and $E_{2}$ respectively, the support of $f$ is restricted to $\left[f_{\min }, f_{\max }\right]$ obtained by minimizing and maximizing with respect to $\mathrm{s}$ respectively:

$$
\begin{gathered}
f_{\min }=E_{1}^{\prime}\left\|\mathbf{h}_{1}\right\|^{4} \quad \text { at } \quad \mathbf{s}=\frac{\mathbf{h}_{1 \perp}^{*}}{\left\|\mathbf{h}_{1 \perp}\right\|} \sqrt{E_{2}} \\
f_{\max }=E^{\prime}\left\|\mathbf{h}_{1}\right\|^{4} \quad \text { at } \quad \mathbf{s}=\frac{\mathbf{h}_{1}^{*}}{\left\|\mathbf{h}_{1}\right\|} \sqrt{E_{2}},
\end{gathered}
$$

where $E_{1}^{\prime}=\left|\frac{\mathbf{h}_{1}}{\left\|\mathbf{h}_{1}\right\|}{ }^{T} \mathbf{v}\right|^{2}, E^{\prime}=E_{1}^{\prime}+E_{2}$, and $\mathbf{h}_{1 \perp}$ is a vector in the null space of $\mathbf{h}_{1}$.

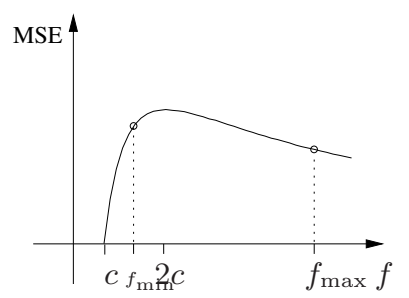

(a) $\operatorname{MSE}(f)$ for $c>0$

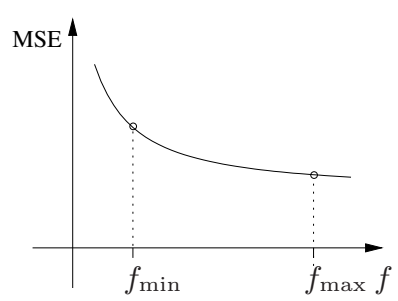

(b) $\operatorname{MSE}(f)$ for $c \leq 0$.
Fig. 2. Typical plots of the MSE as a function of $f$.

Note that from Fig. 2, the $\operatorname{MSE} \mid \mathbf{y}_{1}(f)$ is either, monotonically increasing from $f=0$ to $2 c$ and decreasing from $f=2 c$ to $\infty$ for $c>0$, or strictly decreasing for $c \leq 0$. Since no local minimum exists, the minimum MSE will always occur at the end points of the support of $f$. Therefore, to minimize the MSE, we simply need to compare the MSE values at $f_{\min }$ and $f_{\max }$. The optimal $\mathbf{s}$ is

$$
\begin{aligned}
\mathbf{s}\left(\mathbf{y}_{1}\right)= & \arg \max _{\mathbf{s}}(f) \mathrm{I}\left(\operatorname{MSE}\left(f_{\max }\right) \leq \operatorname{MSE}\left(f_{\min }\right)\right) \\
& +\arg \min _{\mathbf{s}}(f) \mathrm{I}\left(\operatorname{MSE}\left(f_{\min }\right)<\operatorname{MSE}\left(f_{\text {max }}\right)\right)
\end{aligned}
$$

Since $\mathbf{s}=\underset{\left\|\mathbf{h}_{1}\right\|}{\mathbf{h}_{1}^{*}} \sqrt{ } \dddot{E}_{2}$ maximizes $f, \mathbf{s}=\underset{\left\|\mathbf{h}_{1 \perp}^{\prime}\right\|}{\mathbf{h}_{1 \perp}^{*}} \sqrt{ } \dddot{E}_{2}$ minimizes $f$, and $\operatorname{MSE}\left(f_{\max }\right) \leq \operatorname{MSE}\left(f_{\min }\right)$ is equivalent to

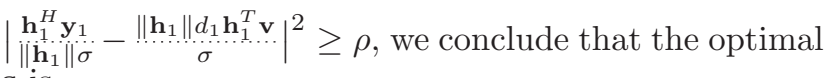
$\mathbf{s}$ is

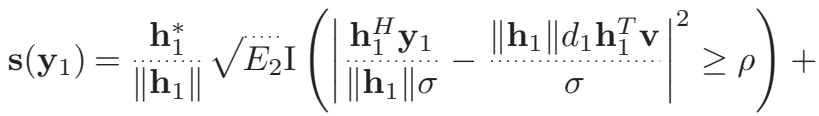

$$
\begin{aligned}
& \underset{\left\|\mathbf{h}_{1 \perp}\right\|}{\mathbf{h}_{1 \perp}^{*}} \sqrt{E_{2} \mathrm{I}} \mathrm{I}\left(\left|\begin{array}{c}
\mathbf{h}_{1}^{H} \mathbf{y}_{1} \\
\left\|\mathbf{h}_{1}\right\| \sigma
\end{array}-\frac{\left\|\mathbf{h}_{1}\right\| d_{1} \mathbf{h}_{1}^{T} \mathbf{v}}{\mid}\right|^{2}<\rho\right),
\end{aligned}
$$

where $\rho=\ldots \ldots E_{1}^{\prime} \ldots$ and $\mathrm{I}(\cdot)$ is the indicator function. This solution implies that when the actual realization of the noise along $\mathbf{h}_{1}$ in the first transmission is small enough there is no advantage in using the measurement from the second step. Therefore, we transmit $\mathbf{s} \propto \mathbf{h}_{1 \perp}^{*}$, which makes the overall estimator only a function of the first measurement. When the actual realization of the noise along $\mathbf{h}_{1}$ in the first transmission is not small enough, there is some merit in incorporating the information from the second measurement and therefore we select $\mathbf{s} \propto \mathbf{h}_{1}^{*}$.

In the noiseless case, $\underset{\left\|\mathbf{h}_{1}\right\|}{\mathbf{h}_{1}^{*}} \sqrt{ } E_{2} \propto \mathbf{y}_{1}^{*}$ which means that the optimal signal to send at the second transmission is the noiseless time reversed version of the signal received at the first stage.

Substituting for $\mathbf{s}\left(\mathbf{y}_{1}\right)$ from (7) into (6), we obtain

$$
\begin{aligned}
& \mathrm{MSE} \mid \mathbf{y}_{1}=\frac{\sigma^{2}}{E^{\prime}\left\|\mathbf{h}_{1}\right\|^{4}}\left\{\mathrm{I}\left(|\alpha|^{2} \geq \rho\right)\left(\begin{array}{c}
E_{2} \\
E^{\prime}+\frac{E_{1}^{\prime}|\alpha|^{2}}{E^{\prime}}
\end{array}\right)\right. \\
& \left.+\mathrm{I}\left(|\alpha|^{2}<\rho\right)\left(\begin{array}{c}
E^{\prime}|\alpha|^{2} \\
\cdots E_{1}^{\prime}
\end{array}\right)\right\}
\end{aligned}
$$

where $\alpha \sim \mathcal{C N}(0,1)$. Taking the expectation over $\alpha$, the MSE simplifies to

$$
\begin{aligned}
\mathrm{MSE}= & \underset{E^{\prime}\left\|\mathbf{h}_{1}\right\|^{4}}{\sigma^{2}}\left\{e^{-\rho \frac{1-2 \rho}{1-\rho}}+\frac{\rho(1+\rho)}{1-\rho} e^{-\rho}\right. \\
& \left.+\frac{1-\rho}{\rho}\left(1-e^{-\rho}(1+\rho)\right)\right\} .
\end{aligned}
$$

We know that $E^{\prime}=\left|\underset{\left\|\mathbf{h}_{1}\right\|}{\mathbf{h}_{1}{ }^{T}} \mathbf{v}\right|^{2}+E_{2}$. MSE is minimized when $E^{\prime}$ is maximized which happens when $\mathbf{v}=\mathbf{h}_{\left\|\mathbf{h}_{1}\right\|}^{*} \sqrt{ } E_{1}$. The value of $\rho$ that minimizes the MSE is given by $\rho_{\text {opt }} \approx 0.2831$. This implies that the amount of energy allocated at the first stage is $E_{1} \approx 0.395 E$ and the remaining energy, $E_{2} \approx 0.605 E$ is used at the second stage. The minimum MSE will be given by,

$$
\mathrm{MSE} \approx \underset{\mathrm{SNR}}{1}(0.9283)
$$

where SNR $=\underset{\left\|\mathbf{h}_{1}\right\|^{4} E}{\sigma^{2}}$. Note that when $\rho=1 / 2$, i.e., we use all of the energy in the first transmission, the 
MSE is $1 / \mathrm{SNR}$. This suggests that substantial MSE improvement can be achieved by making the second transmission depend on the measured signal $\mathbf{y}_{1}$.

\section{SIMULATION RESULTS}

We see that it is indeed possible to get a lower MSE using this model. It is also important to note that the optimum value of $\mathbf{s}\left(\mathbf{y}_{1}\right)$ in (7) depends on $d_{1}$ which is unknown. In other words, it may not be possible to always achieve this minimum MSE. However, if we are given some knowledge about the scatter coefficient then it is possible to incorporate this knowledge in making the optimal decision for s. Another useful approach to solving the problem is as follows. Now assume that the optimal solution to $\mathbf{s}$ is of the form,

$$
\begin{aligned}
\mathbf{s}\left(\mathbf{y}_{1}\right)= & \frac{\mathbf{h}_{1}^{*}}{\left\|\mathbf{h}_{1}\right\|} \sqrt{E_{2}} \mathrm{I}\left(\left|\frac{\mathbf{h}_{1}^{H} \mathbf{y}_{1}}{\left\|\mathbf{h}_{1}\right\| \sigma}-\theta\right|^{2} \geq \rho\right) \\
& +\frac{\mathbf{h}_{1 \perp}^{*}}{\left\|\mathbf{h}_{1 \perp}\right\|} \sqrt{E_{2}} \mathrm{I}\left(\left|\frac{\mathbf{h}_{1}^{H} \mathbf{y}_{1}}{\left\|\mathbf{h}_{1}\right\| \sigma}-\theta\right|^{2}<\rho\right),
\end{aligned}
$$

where $\theta$ is an arbitrary chosen number or can be chosen subject to some given knowledge of the scatter coefficient.

Figure 3(a) shows the simulation results of SNR $\times$ MSE for various values of $\theta$ with SNR of $18 \mathrm{~dB}, \rho=$ 0.2831 , and 100000 runs. At the optimal $\theta$, which is $\left\|\mathbf{h}_{1}\right\|^{2} d_{1} \sqrt{E_{1}} / \sigma$, the MSE $\times \mathrm{SNR} \approx 0.9283$. We see that there is a reasonable range of values for $\theta$ for which MSE $\times$ SNR $<1$. Another interesting observation is that if $\theta$ is far away from its optimal value, the performance of the estimator is as good as that of the optimal scheme for a single transmission, i.e., $\mathrm{MSE} \times \mathrm{SNR}=1$.

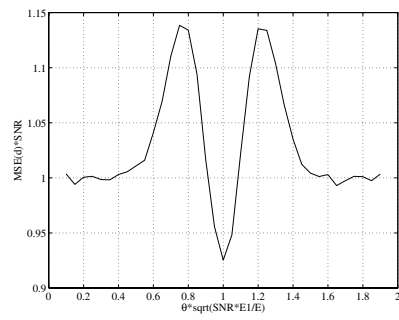

(a)

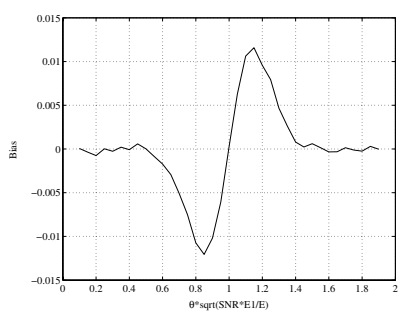

(b)
Fig. 3. SNR $\times$ MSE and Re(bias) vs. $\theta$.

For the same setting as above, we plot the numerical MSE and the bias as a function of $\rho$ and the exact $\operatorname{MSE}(\rho)$ as given in (8) in Fig. 4(a) and 4(b). It is in fact easy to show that the bias of optimal estimator is zero. We observe that the simulation results for the MSE and the bias agree with their analytical equivalents.

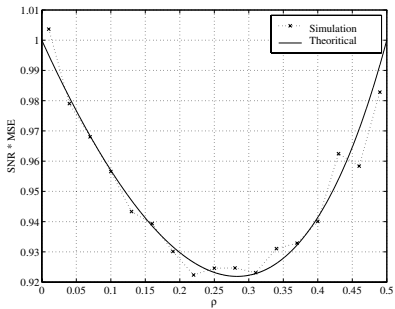

(a)
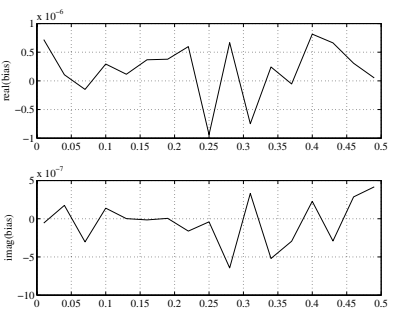

(b)
Fig. 4. SNR $\times$ MSE and bias vs. $\rho$

\section{CONCLUSIONS AND FUTURE WORK}

We assumed a generalized framework for imaging a medium using an array of sensors. We obtained the mean square error for the maximum likelihood estimator of the scattering coefficients. We found the optimal transmission scheme minimizing the MSE and proved that this method can have a distinctive advantage over beamforming methods in a single scatterer environment. Using the MSE, we also found the optimal energy allocation to the two steps involved. Future work would extend our results to a multiple scatterer model. In addition, we intend to generalize this approach from a two step method to an iterative sequence of experiments.

\section{REFERENCES}

[1] E. Kerbrat, C. Prada, and M. Fink, "Ultrasonic nondestructive testing of scattering media using the decomposition of the time-reversal operator," IEEE Trans. on Ultra., Ferro., and Freq., Control, vol. 49, pp. 1103-1112, 2002.

[2] M. Fink, "Time reversal acoustics," Physics Today, pp. 34-40, 1997.

[3] V. V. Fedorov, Theory of Optimal Experiments. New York: Academic Press, 1972.

[4] M. B. Zarrop, Optimal Experiment Design for Dynamic System Identification. New York: SpringerVerlag, 1979.

[5] D. Bliss and A. O. Hero, "Environmental issues for mimo capacity," Signal Processing, vol. 50, no. 9, pp. 2128-2142, 2002.

[6] S. M. Kay, Statistical Estimation. N.J.: PrenticeHall, Englewood-Cliffs, 1991. 